\title{
Measurement of hypersonic attenuation by means of stimulated Brillouin scattering*
}

\author{
P. Leiderer ${ }^{\dagger}$, P. Berberich ${ }^{\ddagger}$, and S. Hunklinger ${ }^{\S}$ \\ Physik Department E 10 der Technischen Universität München, 8046 Garching, Germany
} (Received 19 June 1973; and in final form, 24 July 1973)

\begin{abstract}
A light scattering technique to determine the lifetime of longitudinal phonons in the GHz range is described. The method allows one to measure phonon lifetimes between 5 and $1000 \mathrm{nsec}$ and is applicable to transparent media, in which stimulated Brillouin scattering can be generated.
\end{abstract}

\section{INTRODUCTION}

Most transport properties of liquids and solids can be described in terms of phonons, leading to an increasing interest in methods of investigation of these elementary excitations. For phonons near the origin of the Brillouin zone two methods have proved to be very powerful, namely conventional ultrasonic techniques ${ }^{1,2}$ and light scattering measurements. ${ }^{3}$ The phonon velocities, determined to a high accuracy with either of these techniques, were found to be of the same order of magnitude in most materials. The phonon lifetimes, however, show a much greater diversity, mostly connected with an additional strong dependence on temperature and frequency. The two methods mentioned above are not applicable over the whole broad spectrum of phonon lifetimes, and, in fact, a certain range of lifetimes has until now hardly been accessible with those techniques.

In a typical experiment applying conventional ultrasonic techniques, the sound wave is generated and detected by quartz transducers or vacuum deposited $\mathrm{CdS}$ layers. $\mathrm{Al}$ though well suited for low phonon frequencies and long lifetimes, this technique becomes difficult as the phonon frequency is increased. First, the transducers are required to be parallel within fractions of a wavelength. Second, since the sound absorption usually increases with frequency, the thickness of the sample has to be more and more reduced. This procedure is limited, however, by the condition that the acoustical pulsewidth and the risetime of the electronic system have to be smaller than the time of flight of the phonons. Therefore, conventional ultrasonic techniques are used for the determination of the absolute value of phonon lifetimes which are longer than 500 nsec.

Another very useful method complementing the conventional techniques has become available since the invention of the laser. In this so-called spontaneous Brillouin scattering laser light is scattered by thermal phonons, and the lifetime of these phonons can be inferred from the spectral width of the Brillouin components of the scattered light. In contrast with the transducer technique this method is limited to the measurement of very short lifetimes. The instability of the laser and the limited spectral resolution of the interferometer lead to an instrumental linewidth which can be reduced to about $\Delta v=2 \mathrm{MHz}$ with present techniques $^{4,5}$ corresponding to an upper limit for the lifetime of $\tau=(2 \pi \Delta \nu)^{-1}=80$ nsec. A restriction in the low temperature application of spontaneous Brillouin scattering arises from the decrease with falling temperature of the number of thermal phonons so that it becomes more and more difficult to resolve the Brillouin component from background scattering due to impurities and crystal imperfections. ${ }^{6,7}$

The present paper describes a method allowing the determination of phonon lifetimes from 5 to $1000 \mathrm{nsec}$, thus covering the range of lifetimes inaccessible to conventional ultrasonic techniques and spontaneous Brillouin scattering. This method makes use of the essential advantages of the two other techniques since, on one hand, a constant number of phonons is generated, on the other hand, the decay of the phonons is detected using light scattering.

\section{PRINCIPLE OF MEASUREMENT}

The method is an improvement of the "Two Pulse Method" used by Winterling and Heinicke. ${ }^{8}$ The experimental set-up is shown schematically in Fig. 1. A giant pulse from ruby laser $I$ is focused into the sample with lens $L$ $(f \approx 20 \mathrm{~cm})$ and generates a large number of coherent phonons in the focal volume by stimulated Brillouin scattering (SBS) ${ }^{9,10}$ The phonon frequency is given by

$$
\Delta \nu=2 n v_{s} / \lambda
$$

where $n$ is the refractive index of the sample, $v_{8}$ the sound velocity, and $\lambda$ the laser wavelength. Typical phonon frequencies are $30 \mathrm{GH} z$ for common solids like quartz, but they

FIG. 1. Experimental setup for measuring phonon lifetimes by stimulated Brillouin scattering. Polarization of the giant pulse laser I (indicated by arrows) is in the plane of paper; polarization of laser II is perpendicular to it (indicated by circles). Photocells PH 1 and PH 2 (TRG 105B) record incident and backscattered light, respectively. Registration of the giant pulse light is strongly suppressed, because the beam splitter B is mounted at the Brewster angle $\alpha_{B}$.

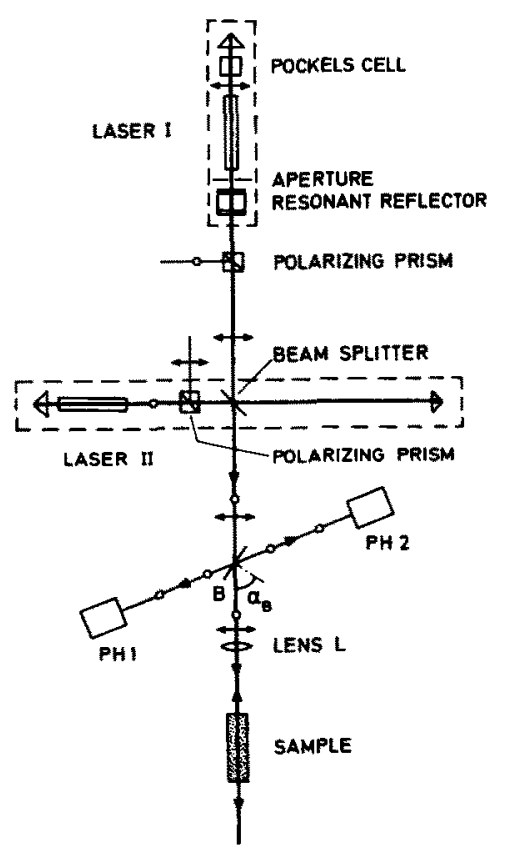




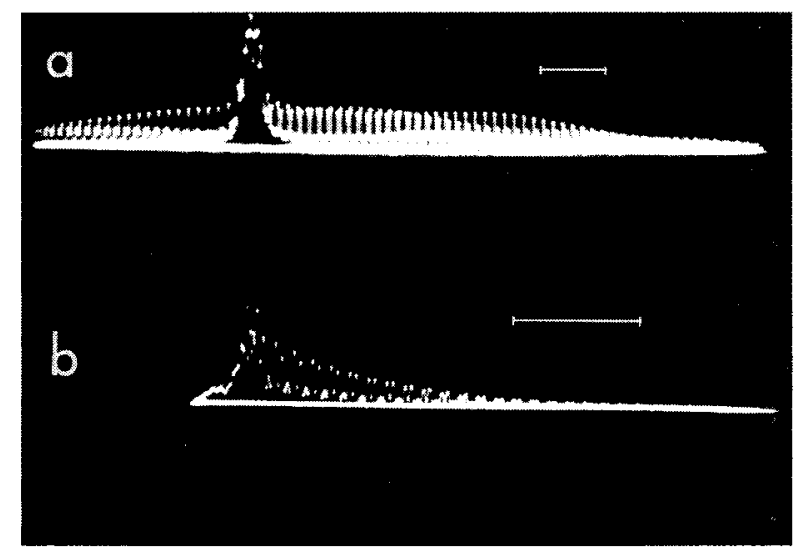

FIG. 2. (a) Typical incident light intensity recorded by photocell PH 1 and a fast oscilloscope (Tektronix 519). The giant pulse is suppressed by a factor of 500. The long regularly spiked train of pulses is due to the second laser. (b) Backscattered light intensity, recorded by photocell $\mathrm{PH}$ 2. The sensitivity of PH 2 is two orders of magnitude higher than of $\mathrm{PH}$ 1. The bar indicates a time interval of $50 \mathrm{nsec}$ in both cases.

can be as low as $0.7 \mathrm{GHz}$, as in liquid helium because of its small velocity of sound.

Light from a second ruby laser (laser II) is used as test light and directed into the light path of laser I with a beam splitter. After generation of the hypersonic phonons in the focal volume, the test light is backscattered by these phonons. If the test light intensity is sufficiently weak that it does not disturb the phonon distribution-of the order of $1 \%$ of laser $\mathrm{I}$ - the backscattered intensity is proportional to the number of phonons still present in the focal volume. As the number of phonons decays, the backscattered intensity decreases with time. Thus from the time dependent ratio of backscattered light to incident light the absolute value of the mean lifetime of the phonons can be obtained.

Similar light probing techniques were developed by Woolf et $a l .{ }^{11}$ and Wilkinson et $a l .^{12}$ who created the hypersonic phonons piezoelectrically. Compared to these methods the very simple scattering geometry of our experiment should be noted.

\section{THE OPTICAL ARRANGEMENT}

Laser $I$ in Fig. 1 is a Q-switched ruby laser with an output power of $10 \mathrm{MW}$ and a pulsewidth of $20 \mathrm{nsec}$. The second ruby laser which generates the probing light has no Q-switch in its resonator. In this mode of operation it produces many



FIG. 3. Ratio of backscattered to incident light $I_{b} / I_{i}$, plotted for the intensities of Fig. 2. light pulses with a typical power of $1-10 \mathrm{~kW}$ and a length of $0.5 \mu \mathrm{sec}$ at an interval of several microseconds. The synchronization of the two lasers is achieved as follows: As soon as photocell PH 1 registers the onset of the first of these test light pulses it triggers the electrooptical Q-switch (Pockels cell) in laser I. After about $150 \mathrm{nsec}$ the giant pulse has developed and generates the phonons in the sample, while the test pulse still has an appreciable intensity.

In the optical arrangement of Fig. 1 the polarization of laser I (indicated by arrows) is in the plane of paper, the polarization of laser II is perpendicular to it (indicated by circles). Figure 2(a) shows a typical oscilloscope trace of the incidentl ight registered by photocell $\mathrm{PH} 1$. The giant pulse is strongly suppressed because its plane of polarization is perpendicular to the beam splitter B in Fig. 1 which is mounted at the Brewster angle $\alpha_{B}$. The testpulse consists of a train of pulses which are formed by superposition of several modes excited in the laser resonator. ${ }^{13}$ The time interval between peaks is exactly determined by the resonator length and can thus be used as a convenient time standard in determining the decay time.

Figure 2(b) represents the backscattered light power, registered by photocell $\mathrm{PH} 2$, as obtained in liquid helium at $1.67 \mathrm{~K}$. Stimulated backscattering sets in at a time when the incident giant pulse reaches the threshold intensity for SBS. Apparently the intensity of the backscattered light of laser II decreases in time, whereas the incident intensity remains nearly constant during the first $200 \mathrm{nsec}$ after the onset of SBS. In order to obtain a well-defined time mark when the stimulated generation of phonons is terminated, the giant pulse can be truncated, e.g., by a Pockels cell ${ }^{14}$ or by a spark gap, ${ }^{15}$ which is mounted at the common focal point of two lenses $(f=5 \mathrm{~cm})$ in the light path of laser I.

\section{DETERMINATION OF THE PHONON LIFETIME}

In Fig. 3 the ratio of backscattered light to incident light from Fig. 2 is plotted as a function of time in a semilogarithmic plot. It is seen from the straight line that the phonons decay exponentially, and a well-defined phonon lifetime of $49 \pm 3 \mathrm{nsec}$ is extracted for liquid helium at $1.67 \mathrm{~K}$ and saturated vapor pressure. Thus, in contrast with the "Two Pulse" method" the absolute value of the phonon lifetime can be obtained within an accuracy of $5 \%$ from every single laser pulse.

Usually a high acoustic amplitude is generated by SBS. ${ }^{10}$ Therefore the phonon lifetime may be seriously affected by finite amplitude effects (for example, the build-up of a shock wave). In this case the attenuation increases by an order of magnitude or more. ${ }^{17}$ There are, however, some experimental criteria by which nonlinear effects may be excluded: (a) if sound absorption is dominated by nonlinear effects, the intensity decreases proportional to time squared ${ }^{17}$ and therefore the decay is not exponential; (b) the amplitude dependence of the absorption may be studied by varying the intensity and the pulse width of the giant pulse.

For a first test this method was applied to liquid helium. As shown in Fig. 3 the decay of the phonons is exponential so that nonlinear effects may be neglected. Fortunately, in 
superfluid helium the lifetimes of phonons with comparable frequencies are known from several other experiments., 11 Our data agree especially well with the results of Greytak et al. ${ }^{4}$ who used spontaneous Brillouin scattering.

Another check was made in crystalline quartz ( $Z$ cut) at $4.2 \mathrm{~K}$. From ultrasonic measurements ${ }^{18}$ the lifetime of the $29 \mathrm{GHz}$ phonons generated by SBS is expected to be much longer than $0.5 \mu \mathrm{sec}$, which is the duration of the test pulse. From this experiment we can quote $1 \mu \mathrm{sec}$ as a lower limit for the phonon lifetime. Therefore, we expect our method to be applicable for lifetime measurements up to $1 \mu \mathrm{sec}$.

\section{APPLICATIONS}

The method described above can be used in all transparent media for measurement of phonon lifetimes, if SBS can be generated. Since the power threshold for SBS in solids is much higher for transverse waves than for longitudinal waves, usually the lifetimes of the later phonons are studied.

Since the sound wave is not generated by a transducer, neither acoustic matching problems nor stringent conditions for parallelism of the end surfaces appear. In solids the medium has to be of high quality only in a small volume into which the laser light is focused. As the method does not rely on the presence of many thermal phonons, it seems to be well suited for low temperature applications, particularly $f \circ r$ studies in rare gas solids and other crystals with low melting temperature. ${ }^{19}$ Another remarkable property is that the measurement lasts less than $1 \mu \mathrm{sec}$ and is therefore applicable to rapidly varying systems. Furthermore, with high light intensities it should be possible to study finite amplitude effects on the acoustical absorption at high frequencies.
*Work supported by Deutsche Forschungsgemeinschaft.

'Present address: School of Applied and Engineering Physics, Cornell University, Ithaca, N. Y. 14850.

†Supported by Graduierten-Förderungs-Programm.

§Present address: Max-Planck-Institut für Festkörperforschung, B. P. 166 Centre de Tri, F-38 Grenoble, France.

'For a review see, for example, H. J. McSkimin, in Physical Acoustics edited by W. P. Mason (Academic, New York, 1964), Vol. I, p. 272.

${ }^{2}$ For a review see, for example, R. Truell, C. Elbaum, and B. B. Chick, Ultrasonic Methods in Solid State Physics (Academic, New York, 1969).

${ }^{3}$ For a review see, for example, P. A. Fleury, in Physical Acoustics edited by W. P. Mason (Academic, New York, 1970), Vol. VI, p. 1.

${ }^{4}$ R. L. St. Peters, T. J. Greytak, and G. B. Benedek, Opt. Commun. 1, 412 (1970).

${ }^{5}$ G. Winterling, F. S. Holmes, and T. J. Greytak, Phys. Rev. Lett. 30, 427 (1973)

${ }^{6}$ A. S. Pine, in Light Scattering in Solids edited by G. B. Wright (Springer Verlag, New York, 1969), paper G-3.

${ }^{7}$ A. S. Pine, Phys. Rev. 185, 1187 (1969).

${ }^{8}$ G. Winterling and W. Heinicke, Phys. Lett. 27A, 329 (1968).

${ }^{9}$ R. Y. Chiao, C. H. Townes and B. P. Stoicheff, Phys. Rev. Lett. 12, 592 (1964).

${ }^{10}$ For a review see, for example, W. Kaiser and M. Maier, in Laser Handbook edited by F. T. Arecchi and E. O. Schulz-Dubois (North-Holland, Amsterdam, 1972), Vol. 2, p. 1077.

${ }^{11}$ M. A. Woolf, P. M. Platzman, and M, G. Cohen, Phys. Rev. Lett. 17, 294 (1966).

${ }^{12}$ C. D. W. Wilkinson and D. E. Caddes, J. Acoust. Soc. Am. 40, 498 (1966)

${ }^{13}$ H. Statz, G. A. DeMars, and C. L. Tang, J. Appl. Phys. 38, 2212 (1967).

${ }^{14}$ D. von der Linde, O. Bernecker, and A. Laubereau, Opt. Commun. 2, 215 (1970).

${ }^{15}$ A. J. Alcock, C. DeMichelis and M. C. Richardson, IEEE J. Quantum Electron. QE-6, 622 (1970).

${ }^{16}$ V. S. Starunov and I. L. Fabelinskii, Sov, Phys.-Usp. 12, 463 (1970).

${ }^{17}$ R. T. Beyer and S. V. Letcher, Physical Ultrasonics (Academic, New York, 1969), p. 202.

${ }^{18}$ M. F. Lewis and E. Patterson, Phys. Rev. 159, 703 (1967)

${ }^{19}$ P. Leiderer, P. Berberich, S. Hunklinger, and K. Dransfeld, Proc. 13th Int. Conf. on Low Temp. Phys., Boulder, 1972, (to be published). 